8th Alexander Friedmann International Seminar

on Gravitation and Cosmology

International Journal of Modern Physics: Conference Series

Vol. 3 (2011) 497-506

(C) World Scientific Publishing Company

DOI: $10.1142 / \mathrm{S} 2010194511001012$

\title{
CRITICAL STEPS IN DATA ANALYSIS FOR PRECISION CASIMIR FORCE MEASUREMENTS WITH SEMICONDUCTING FILMS
}

\author{
A. A. BANISHEV, CHIA-CHENG CHANG and U. MOHIDEEN \\ Department of Physics and Astronomy, University of California, \\ Riverside, California 92521, USA \\ Umar.Mohideen@ucr.edu \\ Received 30 June 2011 \\ Revised 6 July 2011
}

\begin{abstract}
Some experimental procedures and corresponding results of the precision measurement of the Casimir force between low doped Indium Tin Oxide (ITO) film and gold sphere are described. Measurements were performed using an Atomic Force Microscope in high vacuum. It is shown that the magnitude of the Casimir force decreases after prolonged UV treatment of the ITO film. Some critical data analysis steps such as the correction for the mechanical drift of the sphere-plate system and photodiodes are discussed.
\end{abstract}

Keywords: Casimir effect; Indium Tin Oxide; precision measurements.

PACS numbers: 11.25.Hf, 123.1K, 12.20.Fv, 12.20.Ds

\section{Introduction}

The Casimir effect ${ }^{1-6}$ has become important because of its central role in fundamental physics as well as modern technology. Despite its complete quantum nature, with origins in the zero-point energy of a quantized field, the Casimir force is a macroscopic phenomenon. With the recent development of highly sensitive force measurement techniques, it has been demonstrated and measured with unprecedented precision. ${ }^{2-5}$ Because of its unique dependence on the separation and geometry, the Casimir force is expected to play an important role in modern nanoelectro-mechanical systems. ${ }^{7,8}$ The precision measurement of the Casimir force has also been advanced as a new powerful test for hypothetical long-range interactions, supersymmetry, supergravity and string theory. ${ }^{2,5,9}$

The Atomic Force Microscope (AFM) is a surface imaging tool used primarily for mapping the surface topography with atomic resolution. It is based on its sensitivity to small forces acting on the tip of a microfabricated cantilever. It can be adapted to develop new approaches for precision force measurements of the Casimir force between a metal coated sphere and plate. ${ }^{10-15}$ The AFM has become one of the most important tools of choice for precision measurements of the Casimir force 
primarily due to its exquisite force detection sensitivity. The advantages of the AFM technique for Casimir force measurement are described in Ref. 15.

The force detection is based on measuring the deflection of a microfabricated cantilever. An external force will cause an elastic response of the cantilever and the two are related by Hooke's law: $F=k \Delta$, where $k$ is the spring constant of cantilever and $\Delta$ is the deflection distance of cantilever. When the separation distance between the cantilever tip and the sample surface is changed with the movement of the piezoelectric element, the cantilever deflection will correspond to the different forces it experiences at the various separation distances. The deviation of a laser beam reflected off the cantilever tip, measured with photodiodes will record the deflection signals $S_{\text {def }}$ at the various separation distances, leading to a force-distance curve. However, the signal $S_{\text {def }}$ recorded by the photodetector is not in force units but has to be calibrated according to $F=k m S_{\text {def }}$, where $m$ is the cantilever deflection in $\mathrm{nm}$ per unit photodetector signal (sometimes called the optical lever sensitivity) and $k$ is the cantilever spring constant. The product of $m S_{\text {def }}$ is equal to the deflection of the cantilever. If $k^{\prime}=k m$ is known (from the calibration procedure), we can obtain the Casimir force from the deflection signals measured as a function of the sphere plate separation $z$. This deflection signal $S_{\text {def }}$ is calibrated using electrostatic means described. ${ }^{15}$

Here we present some results of measurement the Casimir force between an ITO film and Au sphere before and after UV treatment of the ITO film. We describe some of the critical experimental and data analysis procedures for a precision measurement of the Casimir force in the sphere-plate geometry in general and particularly with regard to semiconductor films. We observe a decrease in the magnitude of the Casimir force after the film undergoes UV treatment.

\section{Experimental Setup and Measurement Procedure}

Detailed information about the cantilever preparation and the experimental technique using the AFM for precision measurements of the Casimir force can be found elsewhere. ${ }^{15}$ The methods for error analysis of the experimental data is presented in Refs. 2, 5 .

In the experiment, the Casimir force was measured between an Au-coated sphere ("Duke Scientific Corp.") of radius $R=101.2 \pm 0.5 \mu \mathrm{m}$ (measured by SEM) attached to conductive AFM cantilever ("Veeco", model MLCT) and ITO film on a quartz plate (prepared by "Thinfilms Inc."). The thickness of gold coating on the sphere was $105 \pm 1 \mathrm{~nm}$, as measured using an AFM. The ITO film (77 nm thickness as measured using ellipsometry and nominal resistivity of $42 \Omega /$ sq) was made by sputter coated on a $1 \mathrm{~cm} \times 1 \mathrm{~cm}$ and $1 \mathrm{~mm}$ thick Quartz plate. The cantilever with the sphere was placed in commercial AFM ("Veeco", modelMultimode V). The ITO sample was mounted on top of a piezo capable of traveling a distance of $2 \mu \mathrm{m}$ between the surfaces. The complete separation distance of the piezo was calibrated using an optical interferometer. ${ }^{16}$ The AFM has been specially adapted to measure the force 
between the test bodies and was placed inside oil free vacuum chamber (working pressure is $10^{-6}$ Torr). To have a low vibration noise environment, we used an optical table and a sand damper box to prevent coupling of the low frequency noise from the mechanical, turbo and cryo pumps. To change the sphere plate distance and avoid piezo drift and creep, a continuous $0.05 \mathrm{~Hz}$ triangular voltage signal was applied to the piezo actuator. To prevent the overheating of the AFM laser in vacuum, leading to instabilities, a liquid nitrogen cooling system was used. We attached a copper braid to the surface of the AFM laser source. The other side of the braid was attached to a liquid nitrogen reservoir, which was also located inside the vacuum chamber. The reservoir could be refilled during the experiment through a liquid feedthrough. During the experiments the temperature was maintained at $2{ }^{\circ} \mathrm{C}$. The cooling of the laser, significantly improves the laser stability, spot size and drift .This made it possible to reduce random error in the Casimir force measurements.

To make measurements of the electrostatic force, the ITO plate is connected to voltage supply (33120A, "Agilent Inc.") operating with $1 \mu \mathrm{V}$ resolution. A $1 \mathrm{k} \Omega$ resistor is connected in series with the voltage supply to prevent surge currents and protect the sample surface during sphere-plate contact. Various voltages in the range from -260 to $-100 \mathrm{mV}$ were applied to that plate, while the sphere remained grounded. To reduce the electrical noise, care was taken to make Ohmic contacts and eliminate all Schottky barriers, to the ITO plate and Au sphere. To minimize electrical ground loops all the electrical ground connections were unified to the AFM ground.

The total force (electrostatic and Casimir) between the Au sphere and the ITO plate was measured as a function of sphere-plate separation distance. Starting at the maximum separation, the ITO plate was moved towards the Au sphere and the corresponding cantilever deflection was recorded at every $0.2 \mathrm{~nm}$ movement till the ITO plate contacted the sphere. A continuous triangular voltage at $0.05 \mathrm{~Hz}$ was applied to the piezo. The sphere-plate separation $z$ is given by:

$$
z=z_{\text {piezo }}+S_{\text {def }} m+z_{0}
$$

where $z_{\text {piezo }}$ is the movement of the plate due to the piezo, $S_{\text {def }} m$ is the change in separation distance due to cantilever deflection and $z_{0}$ is the average separation on contact, which is non zero due to surface roughness (see Fig. 1 in Ref. 15).

A force acting on the sphere leads to the deflection of the cantilever, which is measured as difference signal $S_{\text {def }}$, between the top and bottom set of photodiodes in Volts. The cantilever deflection corresponding to $S_{\text {def }}$, which is given as $\Delta=m S_{\text {def }}$, where $m$ is the proportionality factor defined earlier. The total force acting on the sphere is given by:

$$
F_{\text {total }}=F_{\text {Cas }}+F_{\text {elec }}=k \Delta=k m S_{\text {def }}
$$

Here, $F_{\text {Cas }}$ is the Casimir force, $F_{\text {elec }}$ is the electrostatic force, corresponding to a constant voltage $V$ applied to the plate while the sphere remains grounded. It is 
given by: ${ }^{2}$

$$
F_{\text {elec }}=X(z)\left[V-V_{0}(z)\right]^{2} .
$$

Here, $V_{0}(z)$ is the residual potential difference, which can be present due to the different work functions of the sphere and plate material or contaminations on the ITO surface, ${ }^{17,18}$ and $X(z)$ is a known function related to the capacitance. ${ }^{2,3}$

To reduce random error the total force (the sum of the electrostatic and the Casimir) for each voltage to the plate (each $V$ ) was measured 10 times. Then the applied voltage $V$ was changed to a new value and the experiment repeated. A total of 10 different voltages were applied to the ITO plate and the corresponding total force measured.

\section{Data Processing and Results}

As can be seen from (2), to convert the cantilever deflection in response to force units $(\mathrm{pN})$ the cantilever calibration constant $k^{\prime}=k m$ needs to be found. For this purpose, the electrostatic force is used as in earlier experiments. ${ }^{19-22}$ Using the same electrostatic force, we also determined ${ }^{15}$ the average separation between the sphere and plate surface on contact $z_{0}$, which is needed for the absolute value of the separation $z$ between the bottom of the gold sphere and the ITO surface as shown in Eq. (1). Experimentally, different dc voltages $V$ are applied to the ITO plate and we measure the total force (2) as function of $V$. The cantilever deflection is fitted to the exact theoretical expression ${ }^{2,21}$ shown for the electrostatic force (3) to determine the cantilever calibration constant $k^{\prime}$, the residual potential $V_{0}$ and contact point $z_{0}$. The details of this fitting procedure are described below.

First, the effect of any mechanical drift of the photodetector system with respect to the cantilever has to be subtracted, using the sensitivity limit of the cantilever. For separation distances larger than $1.7 \mu \mathrm{m}$, the total force between the Au sphere and ITO plate is below the instrumental sensitivity. At these separations, the noise is far greater than the signal and in the absence of systematic errors the signal should average to zero and have no dependence on the sphere-plate separation. Thus any observed linear change in $S_{\text {def }}$ has to be from the mechanical drift of the cantilever-photodiode system. To subtract this systematic drift of $S_{\text {def }}$ the following procedure, first outlined in Ref. 15, was used. The variation of $S_{\text {def }}$ at large separation distances of $1.7-2 \mu \mathrm{m}$ was fit to a straight line. This straight line was subtracted from the measured $S_{\text {def }}$ measured at all sphere-plate separations to contact to correct for the effects of photodiode mechanical drift. After this procedure the mean deflection signal at large distances is zero. This procedure was repeated for all experimental measurements. Next we determined the point of sphere-plate contact and the cantilever deflection coefficient $m$ as described in Refs. 2, 15. This value of $m$ is used to evaluate the change in separation $S_{\text {def }} m$ due to the cantilever deflection. This with Eq. (1) determines the sphere-plate separation $z$ up to the value of $z_{0}$ (which is a constant for the complete set of measurements). It is vital 
that an accurate determination of $m$ be made as otherwise it would lead to systematic errors in the separation distance through Eq. (1) as well as the determination of the cantilever deflection and force through Eq. (2). Errors caused by the inaccurate determination of $\mathrm{m}$ and the consequent errors in the determination of separation $z$ will lead to an apparent systematic dependence of the residual potential $V_{0}$ and the average separation distance on contact $z_{0}$. The accurate determination of $m$ involves both (a) precise determination of the contact point between the sphere and plate, and (b) correction for the mechanical drift of the sphere-plate separation when changes in the contact position are considered. The occurrence of (a) is due to the finite density of data points which are separated by $0.2 \mathrm{~nm}$ of $z_{\text {piezo }}$. Thus if the sphere-plate contact occurs between the recorded data points, a linear interpolation technique has to be used to determine the precise contact. The method to accomplish these corrections is described in detail in Ref. 15. The precise value of $\mathrm{m}$ so determined was $m=104.4 \pm 0.5 \mathrm{~nm}$ per unit deflection signal.

Next, the same electrostatic force measurements are used to determine the residual contact potential $V_{0}(z)$, the cantilever calibration constant $k^{\prime}$, and the average separation on contact $z_{0}$. The total deflection signal from applying dc voltages to the ITO plate is given by: ${ }^{11}$

$$
S_{\mathrm{def}}(z)=\frac{X(z)}{k^{\prime}}\left(V_{i}-V_{0}\right)^{2}+S_{\mathrm{Cas}}, \quad i=1, \ldots, 10
$$

The first term represents the electrostatic force corresponding to the applied voltage $V_{i}$. The parabolic dependence of the signal on the applied voltage $V_{i}$ was used to obtain the residual potential difference $V_{0}(z)$ between the grounded sphere and the plate. A total of 10 different voltages $V_{i}$ was applied to the plate for each set of measurements. The cantilever deflection $S_{\text {def }}$ at every applied voltage $V_{i}$ is first determined at every $1 \mathrm{~nm}$ separation using linear interpolation (note that data was collected at separation distances of around $60 \div 300 \mathrm{~nm}$ ). At each separation distance $z$, the $S_{\text {def }}$ is plotted as a function of $V_{i}$. A $\chi^{2}$-fitting procedure is used to draw parabolas and determine its vertex and curvature. The vertex of the parabola corresponds to $V_{0}$ at that separation $z$. The curvature of the parabola is $\beta(z) \equiv$ $X(z) / k^{\prime}$. Thus this curvature can be fit to the exact theoretical form of to obtain $k^{\prime}$ and the separation on contact $z_{0}$. This is described below. The deflection value at the vertex of the parabola corresponds to the cantilever deflection due to the Casimir force, $S_{\text {Cas }}$.

In the case of precision Casimir force measurements it is very important to check that the residual potential difference is independent of sphere-plate separation distance. The $V_{0}$ values obtained at the different sphere-ITO plate separations is shown in Fig. 1. An important feature to be noticed in figure is the constant average value of $V_{0}$ as a function of the separation. If the $V_{0}$ changes systematically with the sphere-plate separation $z$, it indicates the presence of electrostatic surface impurities, space charge effects and/or electrostatic inhomogenieties on the 

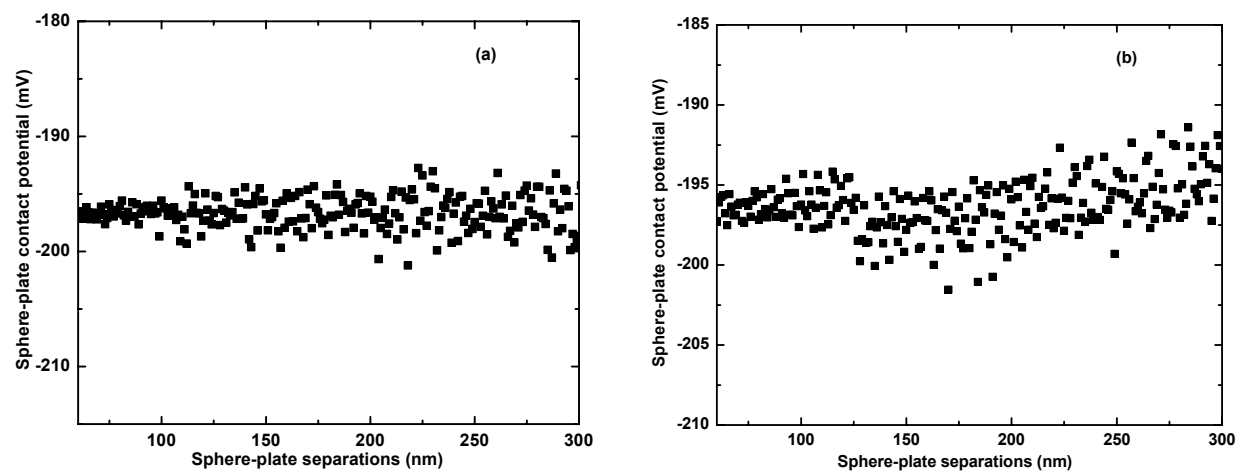

Fig. 1. The residual electrostatic potential between the Au coated sphere and ITO film as a function of the sphere-plate separation, (a) with and (b) without correction of $m$ due to mechanical drift and finite density of data points as described in Ref. 15. The use of the correct value of $m$ leads to a $V_{0}$ which is independent of sphere-plate separation distance as in figure (a). Imprecise evaluation of $m$ leads to a $V_{0}$ which changes with separation as observed in figure (b).

sphere or plate surface. ${ }^{17,18,23}$ In our measurements the $V_{0}$ was found to be independent of separation, varying randomly with no systematic trend, as shown in the Fig. 1(a). The average values of contact potential was found to be equal to $V_{0}=(-196.8 \pm 1.5) \mathrm{mV}$. If the errors in $\mathrm{m}$ due to finite data acquisition rate and mechanical drift of the sphere plate separation are not done, a systematic variation in the residual potential $V_{0}$ is observed as shown in Fig. 1b. An error in m leads to an error in the determination of $\mathrm{z}$ as shown in Eq. (1), which is larger at smaller separations due to the larger $S_{\text {def }}$. The subsequent parabolas generated for the electrostatic force are asymmetric due to the impact of larger $S_{\text {def }}$ at larger applied voltages $V$. This asymmetry leads to an error in the determination by $\chi^{2}$-fitting of the residual potential $V_{0}$ at the vertex of the parabola. This can be observed in Fig. 1(b), where the proper correction in $m$ due to (a) and (b) above has not been done leading to the systematic variation of $V_{0}$ with sphere-plate separation distance.

The value of the parabola curvature $\beta(z)$ depends on both the cantilever calibration constant $k^{\prime}$ and the average separation on contact $z_{0}$. Thus $\beta(z)$ obtained above as a function of the sphere-plate separation $z$, can be used to determine both $z_{0}$ and $k^{\prime}$. By fitting the $\beta(z)$ with theoretical equation for $X(z)$ using the least $\chi^{2}$-fitting as described in Ref. 15 we determined the $k^{\prime}$ and $z_{0}$ values. These values of $k^{\prime}$ and $z_{0}$ so determined, have to be checked and confirmed to be independent of the sphere-plate separation $z$. A special fitting procedure was followed to make this test. The parabola curvature $\beta(z)$ was first fit from a starting point of $60 \mathrm{~nm}$ to an endpoint of $1000 \mathrm{~nm}$ and the values of $k^{\prime}$ and $z^{\prime}$ determined using the least $\chi^{2}$-procedure. Next, the endpoint was decreased from $1000 \mathrm{~nm}$ to $900 \mathrm{~nm}$ and the fitting procedure repeated and corresponding new values of $k^{\prime}$ and $z_{0}$ determined. This 

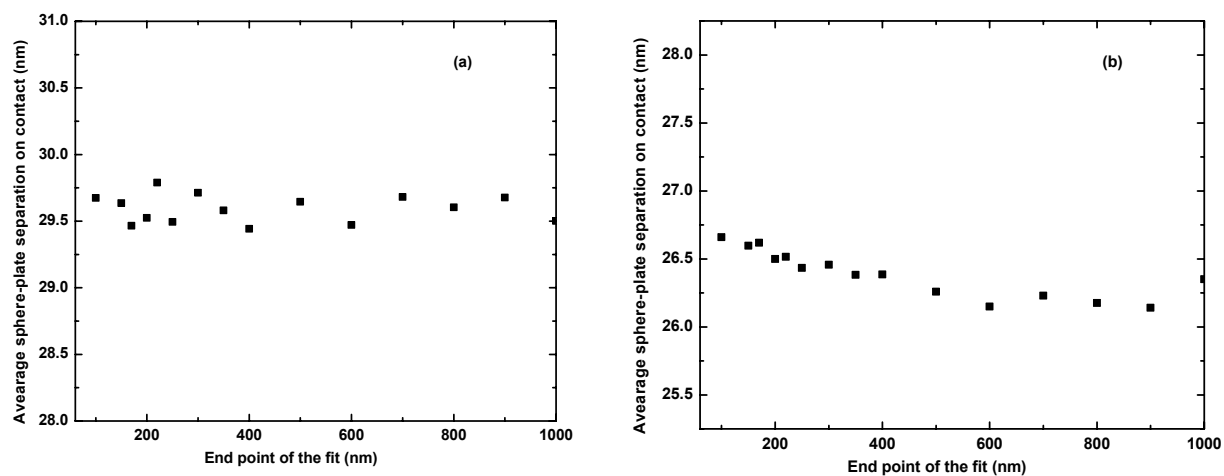

Fig. 2. The average non zero sphere-plate separation on contact (due to roughness) from the measured electrostatic force as a function of the end point of the fit, (a) with, and (b) without proper correction of $m$ due to mechanical drift and finite density of data points. The resulting systematic variation of $z_{0}$ with the change in the endpoint separation from using an inaccurate value of $m$ is shown in figure (b).

procedure was repeated in smaller steps for endpoints below $300 \mathrm{~nm}$. The obtained values of $z_{0}$ and $k^{\prime}$ are shown as a function of the endpoint in Figs. 2(a) and 3, respectively. As can be observed in the figures, the values do not show any systematic dependence on the sphere-plate separation distance. The mean value of $k^{\prime}$ and $z_{0}$ determined were $29.5 \pm 0.4 \mathrm{~nm}$ and $1.51 \pm 0.06 \mathrm{pN} / \mathrm{mV}$, respectively. Failure to determine $m$ precisely, due to the finite density of data points and mechanical drift will lead to systematic errors in $z_{0}$. The resulting systematic variation of the $z_{0}$ with the change in the endpoint separation from using inaccurate values of $m$ is shown in Fig. 2(b). The origins of this systematic error are the same as that discussed in the case of $V_{0}$. Once the determination of the calibration constant $k^{\prime}$ is made we

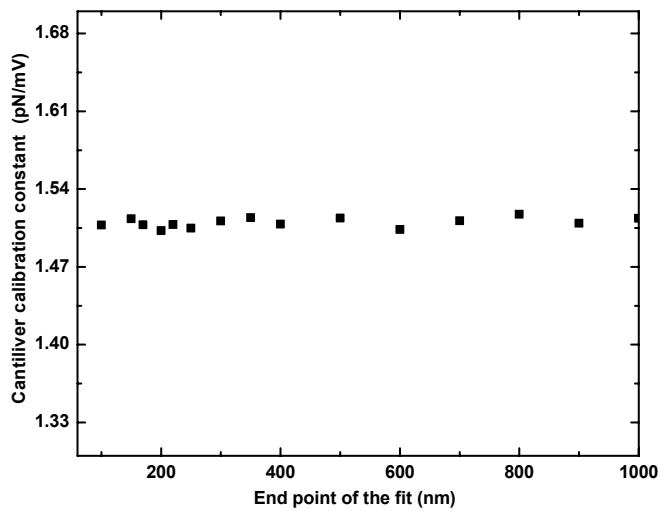

Fig. 3. The cantilever calibration constant $k^{\prime}$ as a function of the end point of the fit. 
can convert deflection signals $S_{\text {Cas }} \rightarrow F_{\text {total }}$, according to $F_{\text {Cas }}=S_{\text {Cas }} k^{\prime}$. Similarly, from the mean value of the average separation on contact $z_{0}$ from Fig. 2(a), the absolute separation $z$ can be determined using Eq. (1).

We compared the above method of determination of the Casimir force to that obtained from the subtraction of the theoretical electrostatic force from the measured total force for each applied voltage $V_{i}$. From the known $k^{\prime}$, we can convert $S_{\text {def }}$ to $F_{\text {total }}$ according to $F_{\text {total }}=S_{\text {def }} k^{\prime}$. The theoretical electrostatic force corresponding to the applied voltage can be subtracted from the total measured force to obtain the Casimir force $F_{\text {Cas }}$ as:

$$
F_{\text {Cas }}^{i}=S_{\text {def }} k^{\prime}-X(z)\left(V_{i}-V_{0}\right)^{2}, \quad i=1, \ldots, 10,
$$

where the $X(z)$ is the expression obtained from the exact theory of the electrostatic force between a sphere and ITO plate. $^{2} \mathrm{~A}$ value of $F_{\text {Cas }}^{i}(z)$ at all $z$ for every $V_{i}$ applied to the plate is obtained. From the set of 10 applied voltages $V_{i}$, the ten $F_{\text {Cas }}^{i}$ are calculated. The experimental measurement of the total force with 10 different applied voltages to the plate was repeated 10 different times for a total of 100 measurements. All 100 Casimir forces are averaged to obtain the mean Casimir force between the Au sphere and ITO plate. The $V_{0}$ in every set of measurement was found to have no systematic dependence on the separation distance. They were all found to vary randomly within the same $2.5 \mathrm{mV}$ range. The average value of $V_{0}$ from the 10 sets of measurements $\left\langle V_{0}\right\rangle$ was used for the subtraction of the electrostatic force as shown in Eq. (5). The two methods of finding the Casimir force were found to lead to the same results. In Fig. 4 the mean Casimir force from the 100 measurements as a function of separation is shown as crosses. For comparison the Casimir force between an Au coated sphere and Au plate is shown as squares. The use of the ITO plate leads to a reduction of the Casimir force by a factor of 1.74,

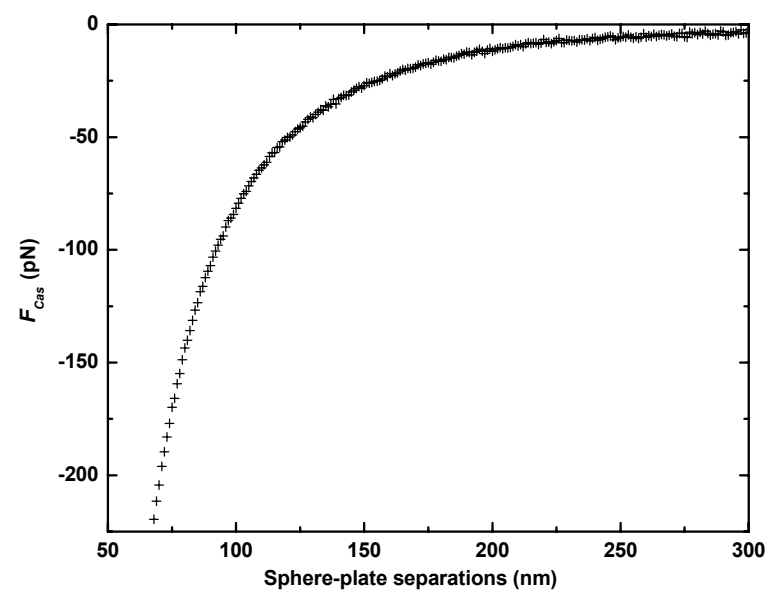

Fig. 4. The mean Casimir force from 100 measurements of the total force (electrostatic + Casimir force) between the ITO film and Au sphere is shown as crosses. 
1.65 and 1.69 at 85, 100 and $120 \mathrm{~nm}$ separations respectively. The UV treatment of the ITO film was found to further reduce the Casimir force.

\section{Conclusions}

We have reported the measurement and procedures for the data analysis of the experimental parameters required for a precision measurement of the Casimir force using an AFM between an ITO film and gold sphere. In comparison with that between an $\mathrm{Au}$ sphere and $\mathrm{Au}$ plate, the Casimir force between a Au sphere and ITO plate was reduced by a factor of 1.65 at $100 \mathrm{~nm}$ surface separtions. When the ITO plate was irradiated with UV light, the Casimir force was observed to be reduce further by a factor of 1.47 . These experiments are still in progress and will be discussed in detail in a forthcoming publication. The results presented here will find important application in nanotechnology, where the Casimir force between metal coated moving elements have been found to be serious limitation on device function.

\section{Acknowledgments}

This work was supported by the DARPA Grant under contract No.S-000354 (equipment, data acquisition, A.A.B., U.M.), NSF Grant No. PHY0970161(analysis, calibration, C-C.C., U.M.) and DOE Grant No. DEF010204ER46131 (analysis, U.M.). The authors wish to thank V. M. Mostepanenko and G. L. Klimchitskaya for their help with the analysis.

\section{References}

1. H. B. G. Casimir, Proc. K. Ned. Akad. Wet. B 51, 793 (1948).

2. M. Bordag, G. L. Klimchitskaya, U. Mohideen and V. M. Mostepanenko, Advances in the Casimir Effect (Oxford University Press, Oxford, 2009).

3. G. L. Klimchitskaya, U. Mohideen and V. M. Mostepanenko, Rev. Mod. Phys. 81, 1827 (2009).

4. G. L. Klimchitskaya, U. Mohideen and V. M. Mostepatenko, Int. J. Mod. Phys. B 25, 171 (2011).

5. R. S. Decca, D. López, E. Fischbach, G. L. Klimchitskaya, D .E. Krause and V. M. Mostepanenko, Ann. Phys. (N.Y.)318, 37 (2005).

6. P. W. Milonni, The Quantum Vacuum. An Introduction to Quantum Electrodynamics (Academic Press, San Diego, 1994).

7. F. Serry, D. Walliser and G. J. Maclay, J. Appl. Phys. 84, 2501 (1998).

8. H. B. Chan, V. A. Aksyuk, R. N. Kleiman, D. J. Bishop and F. Capasso, Science 291, 1941 (2001).

9. V. B. Bezerra, G. L. Klimchitskaya, V. M. Mostepanenko and C. Romero, Phys. Rev. D 81, 055003 (2010).

10. U. Mohideen and A. Roy, Phys. Rev. Lett. 81, 4549 (1998).

11. B. W. Harris, F. Chen and U. Mohideen, Phys. Rev. A 62, 052109 (2000).

12. G. Jourdan, A. Lambrecht, F. Comin and J. Chevrier, Europhys. Lett. 85, 31001 (2009).

13. S. de Man, K. Heeck, R. J. Wijngaarden and D. Iannuzzi, Phys. Rev. Lett. 103, 040402 (2009). 
14. P. J. van Zwol, V. B. Svetovoy and G. Palasantzas, Phys. Rev. B 80, 235401 (2009).

15. H.-C. Chiu, C.-C. Chang, R. Castillo-Garza, F. Chen and U. Mohideen, J. Phys.: Math. Theor. A 41, 164022 (2008).

16. F. Chen and U. Mohideen, Rev. Sci. Instrum. 72, 3100 (2001).

17. W. J. Kim, A. O. Sushkov, D. A. R. Dalvit and S. K. Lamoreaux, Phys. Rev. A 81, 022505 (2010).

18. C. C. Speake and C. Trenkel, Phys. Rev. Lett. 90, 160403 (2003).

19. M. Bordag, U. Mohideen and V. M. Mostepanenko, Phys. Rep. 303, 1 (2001).

20. F. Chen, U. Mohideen, G. L. Klimchitskaya and V. M. Mostepanenko, Phys. Rev. A 72, 020101(R) (2005).

21. F. Chen, U. Mohideen, G. L. Klimchitskaya and V. M. Mostepanenko, Phys. Rev. A 74, 022103 (2006).

22. F. Chen, G. L. Klimchitskaya, V. M. Mostepanenko and U. Mohideen, Phys. Rev. Lett. 97, 170402 (2006).

23. L. Bingqian, Z. Changchun and L. J. Junhua, Micromech. Microeng. 9, 319 (1999). 